\title{
Alternative derivation of the Pegg-Barnett phase operator
}

\author{
A. Luis and L.L. Sánchez-Soto \\ Departamento de Optica, Facultad de Ciencias Físicas, Universidad Complutense, 28040 Madrid, Spain
}

(Received 20 July 1992)

\begin{abstract}
An alternative derivation of the Pegg-Barnett phase operator is presented. This approach is based on the properties of the representation in quantum mechanics of a nonlinear nonbijective canonical transformation. It does not use as its starting point either a finite-dimensional space or the definition of phase states. The features of this formalism are analyzed in terms of this transformation.
\end{abstract}

PACS number(s): 42.50.Dv, 42.50.Ar, 03.65.-w

\section{INTRODUCTION}

The correct definition of phase in quantum mechanics encounters basic difficulties, becoming a subject of continuous investigation from the beginning of the quantum theory until the present time [1]. The relevance of such a variable has stimulated an active search for a satisfactory quantum description as close as possible to its desirable properties.

Two approaches have found great acceptance. In his original work on the quantized electromagnetic field, Dirac [2] proposed the existence of a phase operator that would be defined from a polar decomposition of the annihilation operator in terms of the square root of the number and the exponential of the phase operators [3]. Such exponential of phase, known as the Susskind-Glogower (SG) operator [4], is not unitary and does not allow the existence of a Hermitian phase operator. This formalism exhibits phaselike properties for fields with high average excitation number, and its non-normalizable eigenstates are often considered as states with well-defined phase [5]. However, when the existence of the vacuum becomes significant, the peculiar effects associated with the nonunitarity become apparent.

The Pegg-Barnett (PB) formalism [6], which has been widely employed recently, starts with states of welldefined phase. They ascribe the obstacles to defining a satisfactory phase operator to the use of an infinite Hilbert space from the start, and propose to work with a finite, but arbitrarily large, Hilbert space of $s+1$ dimensions. With the aid of the phase states they are able to define a Hermitian phase operator. Then, after measurable quantities are calculated, the limit as $s$ tends to infinity is taken. This first step of working entirely with a finite-dimensional space before finding the limit is a departure from the usually accepted prescription.

In this work, we present a different derivation of this formalism that does not begin with either a finitedimensional space or the definition of phase states. The idea is to consider a canonical transformation to action and phase-angle variables for a harmonic oscillator [7], and incorporate in this way a finite-dimensional space to the description of the system.

\section{PEGG-BARNETT PHASE OPERATOR}

The PB formalism starts from a previous definition of phase states as

$$
|\theta\rangle=\lim _{s \rightarrow \infty} \frac{1}{\sqrt{s+1}} \sum_{n=0}^{s} e^{i n \theta}|n\rangle,
$$

together with the assertion that the failure to obtain a well-behaved phase operator results from prematurely replacing $s$ by $\infty$ in the summation. Their idea is to work in a finite-dimensional space $\Psi$, spanned by the number states $|n\rangle$, where $n=0, \ldots, s$. The phase states in $\Psi$

$$
|\theta\rangle=\frac{1}{\sqrt{s+1}} \sum_{n=0}^{s} e^{i n \theta}|n\rangle
$$

are then an overcomplete set. A Hermitian operator $\phi$ is constructed, selecting a subset of these states to form a complete orthogonal basis, as

$$
\begin{aligned}
\phi & =\sum_{m=0}^{s} \theta_{m}\left|\theta_{m}\right\rangle\left\langle\theta_{m}\right|, \\
\theta_{m} & =\theta_{0}+\frac{2 \pi}{s+1} m,
\end{aligned}
$$

where $\theta_{0}$ is an arbitrary reference phase. Choosing the basis beginning with $\theta_{0}$ to span the space $\Psi$ corresponds to the classical procedure of choosing a particular $2 \pi$ window in which to express the value of $\arctan (p / q)$.

A unitary operator exponential of the phase is then

$$
e^{i \phi}=\sum_{n=1}^{s}|n-1\rangle\left\langle n\left|+e^{i(s+1) \theta_{0}}\right| s\right\rangle\langle 0| .
$$

The annihilation operator on $\Psi$ is defined via its polar decomposition in terms of the square root of the number and $e^{i \phi}$ operators, obtaining [8]

$$
a=\sum_{n=1}^{s} \sqrt{n}|n-1\rangle\langle n|,
$$

which gives a commutation relation

$$
\left[a, a^{\dagger}\right]=I-(s+1)|s\rangle\langle s| .
$$

The difficulties that the SG formalism has in describing the vacuum are overcome by working with $\Psi$. However, the special role played by the upper state $|s\rangle$ in removing these difficulties also precludes taking the infinite $s$ limit of the above operator, because the difficulties would 
then reappear. Pegg and Barnett have never claimed to have established a well-defined Hermitian operator in the infinite-dimensional space $\mathcal{H}$. Their procedure must be worked entirely in the $\Psi$ space, and allows its dimension tend to infinity only after expectation values are calculated in order to become true expectation values. We are not going to discuss the consistency of the theory, but just mention that there may appear to be difficulties in general because of the finiteness of the space means that some operators, such as $a$, have different properties from those of their infinite-space counterparts. Pegg and Barnett emphasize that such differences will not lead to detectable physical differences when the limit is eventually taken [9].

Then, while on the one hand the finiteness of the state space is crucial to the existence of the phase operator, on the other, the infinite limit is necessary both to obtain the final results and to overcome the undesirable terms depending on the upper state. In fact, one of the successes of this approach, the characterization of the vacuum as a random phase state, is obtained only in the limit $s \rightarrow \infty$, and only then an arbitrary phase state $|\theta\rangle$ can be considered as having a well-defined phase irrespective of the phase reference $\theta_{0}$.

Nevertheless, it can be advantageous to interpret Pegg and Barnett's statement about the impossibility of distinguishing, by physical experiments, the differences between an infinite and a finite but arbitrarily large dimensional space in the sense that one can describe a given physical system as accurately as desired by its projection on a finite-dimensional space with a proper choice of its dimension. This is equivalent to say that, for a physically accessible or preparable state (prepared from the vacuum by interactions for a finite time and a finite energy exchange), one can always choose a value of $s$ so that the physical effects of this cutoff are as small as desired. This does not mean, however, that $s$ is a fixed number whose choice depends on the state of the system. Instead, $s$ is a parameter which tends to infinity. In this limit, the difference in the physical properties of any physically accessible state of the infinite Hilbert space and its $\Psi$ space counterpart vanishes.

The PB approach requires the infinite $s$ limit to be taken of $s$-dependent expectation values, which have the general form

$$
\langle f(\phi)\rangle_{\psi}(s)=\sum_{m=0}^{s} f\left(\theta_{m}\right)\left|\left\langle\theta_{m} \mid \psi\right\rangle\right|^{2}
$$

Two ways to do this are as follows. First, one could give an expression for $|\psi\rangle$ in the $\Psi$ space, carry out the summation, and then take the limit as $s \rightarrow \infty$. This procedure can be difficult, especially the first step, if we are interested in the phase properties of a state which needs the infinite-dimensional space for its normal description. This has been done numerically for a coherent state [10]. A second, and widely used, method is applicable for states, such as physically accessible states, for which $|\langle n \mid \psi\rangle|$ eventually decreases sufficiently rapidly with increasing $n$. For such states the limit of (2.7) can be found without inserting the particular expression for the $\Psi$ space form of $|\psi\rangle$. The result obtained is

$$
\langle f(\phi)\rangle_{\psi}=\int_{\theta_{0}}^{\theta_{0}+2 \pi} d \theta f(\theta) P_{\psi}(\theta)
$$

where the phase probability distribution is given by

$$
P_{\psi}(\theta)=\lim _{s \rightarrow \infty} \frac{s+1}{2 \pi}|\langle\theta \mid \psi\rangle|^{2}=\frac{1}{2 \pi}\left|\sum_{n=0}^{\infty} e^{-i n \theta}\langle n \mid \psi\rangle\right|^{2} \text {. }
$$

Note that the last term is nothing but the projection of the state over a SG phase state

$$
|\vartheta\rangle=\frac{1}{\sqrt{2 \pi}} \sum_{n=0}^{\infty} e^{i n \theta}|n\rangle
$$

Then, what the expectation value (2.8) tells us is that this formalism, at least by this way, corresponds to consider the SG phase states as orthogonal eigenstates of a phase operator. Although it would be a desirable result, it cannot take place. The PB formalism circumvents this problem and arrives at Eq. (2.8) by using, instead of the SG phase states, their orthogonal and normalizable PB counterparts. Phase relations are then defined and phase properties calculated within this context before the limit is taken, in order to avoid dealing with the troublesome nonorthogonality of the SG states which precludes (2.8).

Note that

$$
\lim _{s \rightarrow \infty} \phi=\int_{\theta_{0}}^{\theta_{0}+2 \pi} d \theta|\vartheta\rangle \theta\langle\vartheta|
$$

is the phase operator introduced by Garrison and Wong [11], and independently by Galindo [12], which have many interesting properties. However,

$$
\lim _{s \rightarrow \infty} f(\phi)=\int_{\theta_{0}}^{\theta_{0}+2 \pi} d \theta f(\theta)|\vartheta\rangle\langle\vartheta| \neq f\left(\lim _{s \rightarrow \infty} \phi\right) .
$$

In view of (2.12), the $\mathrm{PB}$ formalism cannot be regarded as an approximation to the formalism involving the Garrison-Wong operator (which, incidentally, satisfies the canonical commutation relation $\left[a^{\dagger} a, \phi\right]=i$ on a dense set of the Hilbert space of state vectors, although approximating even simple physical states by members of said dense set is a very awkward business [13]) because very different results are obtained from the two approaches. Although in the $s \rightarrow \infty$ limit the PB phase and exponential operators approach the Garrison-Wong and Susskind-Glogower operators, respectively, the PB formalism excludes taking these limits because of the inequality in (2.12).

In what follows we give another derivation of this formalism, which starts with the infinite Hilbert space $\mathcal{H}$ and in which the phase operator is not derived from the phase states, in order to make the analysis clearer.

\section{A DIFFERENT DERIVATION OF THE PEGG-BARNETT FORMALISM}

A crucial point in the $\mathrm{PB}$ approach is the use of a finite space. Such a space can be easily incorporated to the 
description of a system in quantum mechanics in place of the cutoff previously discussed.

In a recent work [14], we studied the representation in quantum mechanics of the nonlinear and nonbijective canonical transformation relating a one-dimensional harmonic oscillator of unit frequency with another of frequency $\kappa, \kappa$ being a non-negative integer. This transformation is a dilation in the angle-action variables of the form [7]

$$
j=\kappa \bar{j}, \quad \kappa \phi=\bar{\phi}
$$

where

$$
j=\frac{1}{2}\left(p^{2}+q^{2}\right), \quad \phi=\arctan \left(\frac{p}{q}\right)
$$

are the action and phase-angle variables of the oscillator, and the bared and unbared variables refer to the new and old phase spaces, respectively.

Due to the nonbijectivity [a sector of angle $2 \pi / \kappa$ in the $(q, p)$ plane is mapped on the full $(\bar{q}, \bar{p})$ plane] the $(\bar{j}, \bar{\phi})$ or $(\bar{q}, \bar{p})$ variables cannot be a complete set of coordinates in the new space. Thus, in the quantum case it is necessary to add to the Hilbert space $\overline{\mathcal{H}}$ associated with the $(\bar{q}, \bar{p})$ variables a finite-dimensional space $\mathcal{V}$ to completely describe the physical system.

In this way, a unitary representation for the transformation (3.1) can be given $U: \mathcal{H} \rightarrow \overline{\mathcal{H}} \otimes \mathcal{V}$,

$$
U=\sum_{n=0}^{\infty} \sum_{\lambda=0}^{\kappa-1}|\bar{n}\rangle|\lambda\rangle\langle\kappa n+\lambda|,
$$

where $|\lambda\rangle$ is a basis in $\mathcal{V}$ and $|\bar{n}\rangle$ is the number basis in $\overline{\mathcal{H}}$.

Thus this transformation not only naturally introduces a finite-dimensional space, but involve the phase in a way that we think can be useful in the study of the PB formalism. A similar technique of enlarging the Hilbert space has been employed by Newton [15] in order to find quantum representations of the action-angle variables for the harmonic oscillator.

A crude look at Eq. (3.1) could lead to the interpretation that, in the limit $\kappa \rightarrow \infty$, the discrete $j$ (number of excitations) and the bounded $\phi$ will become a continuous $\bar{j}=j / \kappa$ and an unbounded $\bar{\phi}=\kappa \phi$. The new variables will become then better behaved than their original counterparts with respect to the existence of a phase operator. This is obviously not true, and the new variables are on an equal footing with the original ones, which is the reason for the difficulties for a unitary representation of this transformation. Then, overcoming these difficulties would, at the same time, provide a tool for the description of the phase that is more accurate as $\kappa$ becomes (arbitrarily) higher.

The transformation by $U$ of the number operator

$$
U a^{\dagger} a U^{\dagger}=\kappa \bar{a}^{\dagger} \bar{a}+\sum_{\lambda=0}^{\kappa-1} \lambda|\lambda\rangle\langle\lambda|
$$

splits in two terms acting on $\overline{\mathcal{H}}$ and $\mathcal{V}$, respectively. This expression for the number operator does not seem more adequate than the original one for our purposes if we do not introduce any simplification. As we expect that some kind of advantage can arise in the limit of an arbitrarily high dimension for $\mathcal{V}$, we can exploit this finitedimensional space, focusing our attention momentarily on the projection of the number operator on $\mathcal{V}$, and trying to find the operator corresponding to its conjugate variable. As pointed out by Newton [15], when working in the phase representation the number operator in the enlarged space can be represented by a differential operator that formally coincides with the third component of an angular momentum. So, we take the $\mathcal{V}$ space as carrying a $\kappa$-dimensional unitary irreducible representation of $\mathrm{SU}(2)$ and identify the $|\lambda\rangle$ basis as the eigenvectors of one of its infinitesimal generators

$$
J_{z}|\lambda\rangle=\left(\lambda-\frac{\kappa-1}{2}\right)|\lambda\rangle .
$$

Note that, effectively, the second term on the right-hand side of (3.4) is the third component of an angular momentum.

In this way a unitary operator for the exponential of the phase can be obtained from a polar decomposition of the projection of the angular momentum in the $X Y$ plane in terms of a radial component and an exponential of the phase angle in the form [16]

$$
J_{-}=e^{i \bar{\phi}} J_{r}, \quad J_{+}=J_{r} e^{-i \bar{\phi}},
$$

where

$$
J_{r}=\sqrt{J_{+} J_{-}}
$$

The solution of this equation for a unitary operator $e^{i \bar{\phi}}$ gives

$$
e^{i \bar{\phi}}=\sum_{\lambda=1}^{\kappa-1}|\lambda-1\rangle\left\langle\lambda\left|+e^{i \kappa \theta_{0}}\right| \kappa-1\right\rangle\langle 0|,
$$

$\kappa \theta_{0}$ being an arbitrary phase. Taking $s+1=\kappa$, this operator coincides with (2.4) except for the space of definition. From this operator all the PB formalism can be derived now in the $\mathcal{V}$ space, the phase states basis in $\mathcal{V}$ being

$$
\left|\bar{\theta}_{m}\right\rangle=\frac{1}{\sqrt{\kappa}} \sum_{\lambda=0}^{\kappa-1} e^{i \lambda \theta_{m}}|\lambda\rangle .
$$

These results can be translated into the $\mathcal{H}$ space by means of the transformation $U$. The PB formalism, in our language, consists in identifying the finitedimensional space $\Psi$ with $U^{\dagger}(|\overline{0}\rangle \otimes \mathcal{V})$. A basis in $\mathcal{H}$ can be given as

$$
\left|n, \theta_{m}\right\rangle=U^{\dagger}\left(|\bar{n}\rangle\left|\bar{\theta}_{m}\right\rangle\right),
$$

and the PB phase states will be now $\left|0, \theta_{m}\right\rangle$.

This approach cannot be satisfactory yet. In the first place, we have derived this formalism in the infinitedimensional space $\mathcal{H}$ and, as a consequence, we obtain infinite many copies, labeled by $n$, of the phase states and phase operator. In the second place, we have attempted to describe the phase only in terms of the $\mathcal{V}$ 
space, and $\overline{\mathcal{H}}$ remains merely as a spectator.

When we consider the limit of high $\kappa$ and restrict ourselves to the physically accessible states as defined by Pegg and Barnett $[6,9]$ in their approach, the number operator can be replaced by the second term in Eq. (3.4). This corresponds to neglecting the role of the $\overline{\mathcal{H}}$ space and, together with this, taking just the first copy of the $\mathcal{V}$ space in $\mathcal{H}$ (the one spanned by $\left|0, \theta_{m}\right\rangle$ ) as a closer description of a state.

Note that the rest of variables (like the annihilation operator) remain, in this derivation, always in their usual form; they do not need this procedure to be defined and it does not affect them. Thus, by a different route, we have arrived at the same description of phase as we discussed in Sec. II, except that here the physical properties, such as expectation values, depend on $\kappa$ rather than $s$. When the appropriate limit is taken, the description will approach that which is expected for phase in quantum mechanics, and which is now widely used.

\section{RELATIONSHIP BETWEEN THE TWO DERIVATIONS}

It is clear that the two approaches of Secs. II and III cannot be too distant. The original derivation assumes as a complete description the one realized in a finitedimensional space. In our approach this corresponds to considering the image by $U$ of the dynamical variables (the annihilation operator) and eliminating the $\overline{\mathcal{H}}$ space.

If we express the annihilation operator in $\overline{\mathcal{H}} \otimes \mathcal{V}$ we have

$$
U a U^{\dagger}=\sum_{\lambda=1}^{\kappa-1} \sqrt{\kappa \bar{n}+\lambda}|\lambda-1\rangle\langle\lambda|+\sqrt{\kappa} \bar{a}| \kappa-1\rangle\langle 0| .
$$

A drastic elimination of the $\overline{\mathcal{H}}$ space to retrieve the $\mathrm{PB}$ derivation can be done taking $\bar{n}=0$ and $\bar{a}=0$, obtaining the annihilation operator (2.5). Now it is possible to obtain the exponential of the phase operator from a polar decomposition of this annihilation operator in the form

$$
a=e^{i \phi} \sqrt{n}
$$

where by $a$ here it must be understood the right-hand side of Eq. (4.1) after the previous comments, and by $n$ the second term on the right-hand side of Eq. (3.4).

This equation has a unitary solution for $e^{i \phi}$ that is the same as that obtained before. Then the Hermitian phase operator can occur from a polar decomposition, due not to the existence of phase states, but to the finite dimension of the space, expressed as well on the fact that the upper state is annihilated by the creation operator

$$
a^{\dagger}|\kappa-1\rangle=0 \text {, }
$$

a condition that must be taken into account when resolving Eq. (4.2).
This elimination of the $\overline{\mathcal{H}}$ space then needs a more subtle setting to recover the well-established results of quantum mechanics. This leads to a possibly strange relation between the $\mathrm{PB}$ formalism and the SG phase states shown at the end of Sec. II. Clearly, in the infinite limit for the dimension, some kind of relation must appear because the PB phase states are a projection on the finite-dimensional space of the SG phase states.

In the context of this work the image of a SG state [17]

$$
\begin{aligned}
U\left(\frac{1}{\sqrt{2 \pi}} \sum_{n=0}^{\infty} e^{i n \theta}|n\rangle\right) \\
\quad=\left(\frac{1}{\sqrt{2 \pi / \kappa}} \sum_{n=0}^{\infty} e^{i n \kappa \theta}|\bar{n}\rangle\right)\left(\frac{1}{\sqrt{\kappa}} \sum_{\lambda=0}^{\kappa-1} e^{i \lambda \theta}|\lambda\rangle\right)
\end{aligned}
$$

factorizes into a SG state in $\overline{\mathcal{H}}$ and a $\mathrm{PB}$ in $\mathcal{V}$. Dismissing now the $\overline{\mathcal{H}}$ space, in the limit $\kappa \rightarrow \infty$ for physically accessible states, allows the orthogonalization of the SG states by the selection of a subset beginning with $\left|\theta_{0}\right\rangle$ as discussed earlier.

The same conclusions can be drawn starting from the expression in $\overline{\mathcal{H}} \otimes \mathcal{V}$ for the $\mathrm{SG}$ operator $E=\sum_{n=1}^{\infty}|n-1\rangle\langle n|$,

$U E U^{\dagger}=I \otimes \sum_{\lambda=1}^{\kappa-1}|\lambda-1\rangle\langle\lambda|+\bar{E} \otimes| \kappa-1\rangle\langle 0|$.

The elimination of the $\overline{\mathcal{H}}$ space by replacing $\bar{E}$ with an arbitrary exponential of phase leads to Eq. (2.4) of the PB formalism.

\section{CONCLUSIONS}

The PB formalism involves the construction of a phase operator in a finite-dimensional space and the identification of states of a well-defined phase. Finally the limit is taken of expectation values obtained from this operator as the dimension of the space is allowed to tend to infinity. We have shown that, with a proper choice of variables, the same phase operator can be derived without the first two steps. This equivalent approach is based on the infinite Hilbert space, but possesses the main features of the PB formalism, including the ones related to limit taking.

\section{ACKNOWLEDGMENT}

We are much indebted to Professor E. Bernabeu for his continual advice and interest in the present work. 
[1] S. M. Barnett and D. T. Pegg, J. Phys. A 19, 3849 (1986).

[2] P. A. M. Dirac, Proc. R. Soc. London Ser. A 114, 243 (1927).

[3] P. Carruthers and M. M. Nieto, Rev. Mod. Phys. 40, 441 (1968).

[4] L. Susskind and J. Glogower, Physics 1, 49 (1964).

[5] J. M. Lévy-Leblond, Ann. Phys. (N.Y.) 101, 319 (1976).

[6] D. T. Pegg and S. M. Barnett, Europhys. Lett. 6, 483 (1988).

[7] R. Dirl, P. Kasperkovitz, and M. Moshinsky, J. Phys. A 21, 1835 (1988).

[8] S. M. Barnett and D. T. Pegg, J. Mod. Opt. 36, 7 (1989).

[9] D. T. Pegg and S. M. Barnett, Phys. Rev. A 43, 2579 (1991).
[10] V. Bužek, A. D. Wilson-Gordon, P. L. Knight, and W. K. Lai, Phys. Rev A 45, 8079 (1992).

[11] J. C. Garrison and J. Wong, J. Math. Phys. 11, 2242 (1970).

[12] A. Galindo, Lett. Math. Phys. 8, 495 (1984); 9, 263 (1985).

[13] J. Bergou and B.G. Englert, Ann. Phys. (N.Y.) 209, 479 (1991).

[14] A. Luis and L. L. Sánchez-Soto, J. Phys. A 24, 2083 (1991).

[15] R. G. Newton, Ann. Phys. (N.Y.) 124, 327 (1980).

[16] A. Vourdas, Phys. Rev. A 41, 1653 (1990).

[17] A. Luis and L.L. Sánchez-Soto, Quantum Opt. (to be published). 